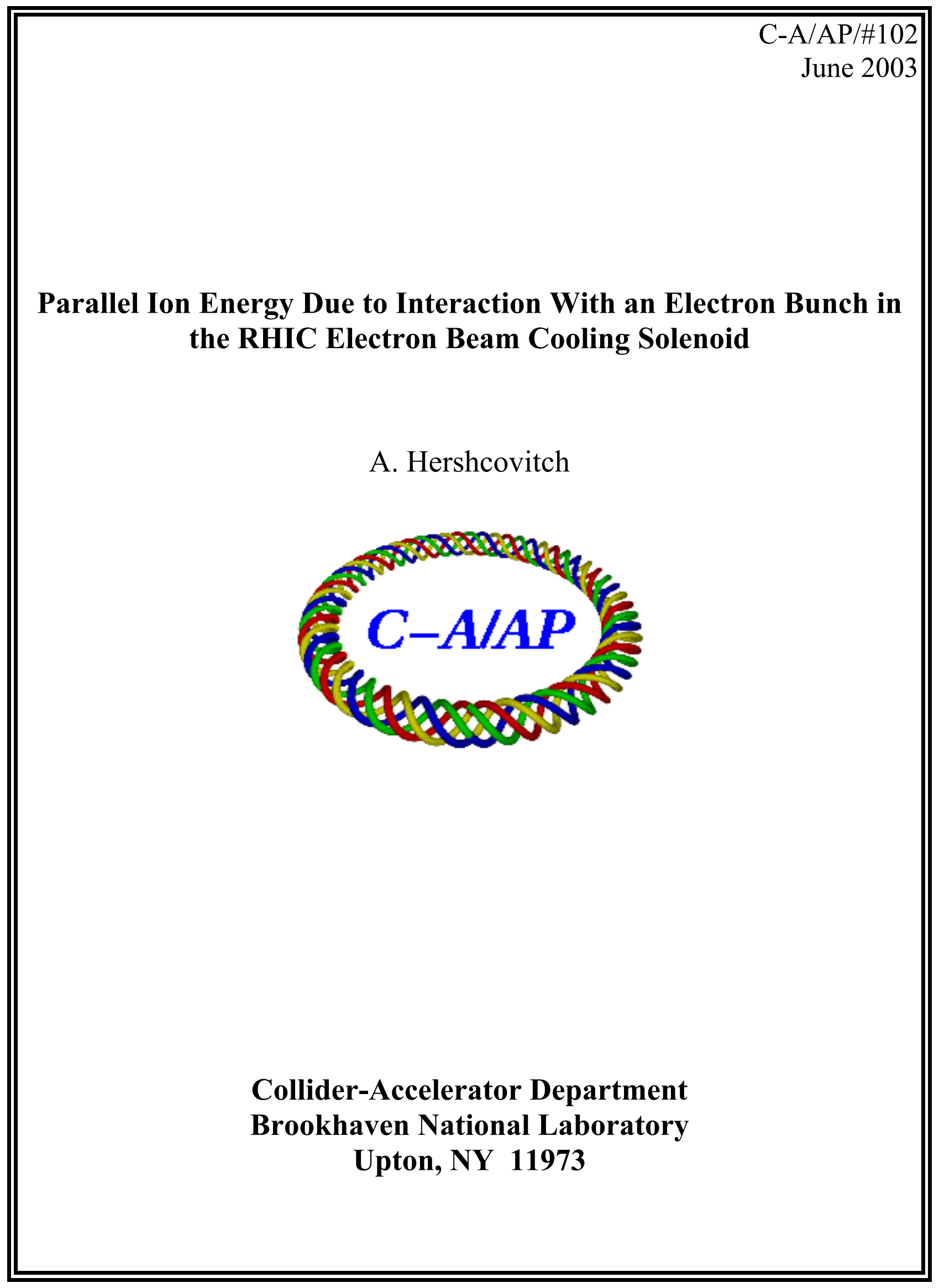




\title{
Parallel Ion Energy Spread Due to Interaction With an Electron Bunch in the RHIC Electron Beam Cooling Solenoid
}

\author{
Ady Hershcovitch
}

Computations designed to evaluate the effect electric fields, generated by an electron bunch, have on ions are performed in this note. Resultant parallel ion energy spread that occurs during interaction in the RHIC electron beam cooling solenoid is found to be negligible. Furthermore, compared to intrinsic accelerator generated energy spread that electron bunch driven parallel ion energy spread is infinitesimal.

\section{Introduction}

Under present RHIC electron beam cooling scenario, electron and ion bunches are to interact in a 30 -meter long solenoid. Transverse ion temperature is to be reduced by collisions with low temperature electrons. Ions and electron move with an identical drift velocity. But the electron bunch, which is rather dense, is an order of magnitude shorter than the ion bunch. Pictorially (not to scale), ions and electrons in the bunch rest frame are shown in figure 1 below.

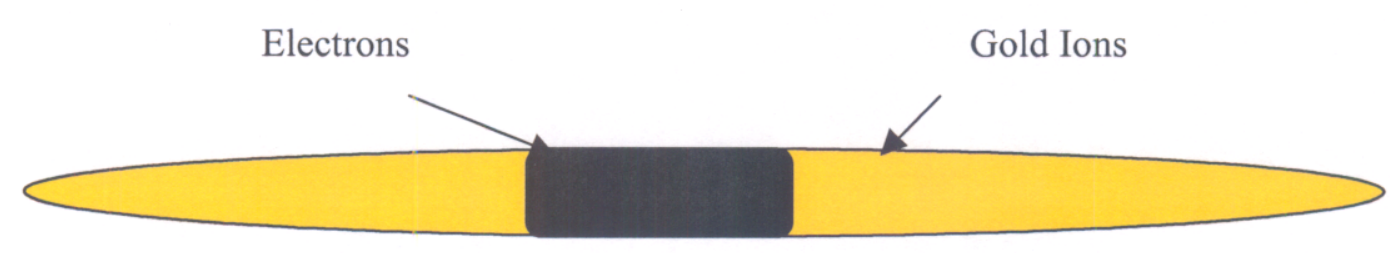

Figure 1

Hence, ions in the bunch, especially those at the electron bunch edge are subjected to an intense electric field. Those at electron bunch front will be decelerated, while ions at the tail end will be accelerated. Additionally, due to ion motion relative to the electron bunch during the interaction period some ions undergo energy change as they move through an electric potential generated by the electron bunch. While this effect cannot result in net 
energy exchange between ion and electrons due to the conservative nature of the interaction, it could result in larger ion energy spread. In this note, parallel ion energy spread due to these effects is evaluated.

Parameters relevant to the calculations are: total electron bunch charge $\mathrm{Q}_{\mathrm{e}}=10 \mathrm{nC}$. In the lab frame, interaction time $\tau_{1}$ is

$$
\tau_{l}=\frac{30 \text { meter }}{3 \times 10^{8} \text { meter } / \mathrm{sec}}=10^{-7} \mathrm{sec}
$$

Ion bunch length $=30 \mathrm{~cm}$

Electron bunch length $=3 \mathrm{~cm}$

And, bunch radii $r_{i}=r_{e}=2 \mathrm{~mm}$.

Computations are next performed in the bunch rest frame. Since $\gamma=100$, ion bunch lehgth is 30 meters and electron bunch length is 3 meters in the bunch rest frame of reference. The interaction time $\tau$ in this frame is $\tau=\tau 1 / \gamma=10^{-9} \mathrm{sec}$.

\section{Ion Energy Change Resulting From Electron Bunch Attraction}

Ion energy gain (or loss) can be evaluated from change in ion velocity, which can be determined from the force $\mathrm{F}$ exerted on an ion by the electron bunch and the interaction time $\tau$.

Change in an ion velocity $\Delta \mathrm{v}$ is

$$
\Delta v=\frac{F}{m_{i}} \tau
$$

But $F=q_{i} E_{e b}$. Hence,

$$
\Delta \nu=q_{i} \frac{E_{e b}}{m_{i}} \tau
$$

Where $E_{e b}$ is the electric field due to the electron bunch at ion location; $q_{i}$ and $m_{i}$ are the ion mass and charge respectively.

In MKS units $\mathrm{q}_{\mathrm{i}}$ and $\mathrm{m}_{\mathrm{i}}$ are $1.264 \times 10^{-17} \mathrm{C}$ and $3.27 \times 10^{-22} \mathrm{~kg}$ respectively.

Calculating energy change is trivial once the electric $\mathrm{E}_{\mathrm{eb}}$ is known. Use can be made of an expression for the electric field that can be found in Sacherer ${ }^{1}$, since the electron bunch 
has ellipsoidal symmetry. That expression was derived from an equation that gives the potential of a charged ellipsoid, which can be found in Kellogg's book. ${ }^{2}$ Therefore, since the electron bunch can be approximated by an ellipsoid of dimension $a, b$, and $c, E_{e b}$ along the long axis (direction of motion) $\mathrm{x}$ (from the bunch center) is given by,

$$
E_{e b}=2 \pi e a b c x \int_{0}^{\infty} \frac{n(T) d s}{\left(a^{2}+s\right)^{3 / 2} \sqrt{b^{2}+s} \sqrt{c^{2}+s}}
$$

Where the integration ds is over a surface element, e is the elementary charge, and $n(T)$ is the charge distribution function. Assuming that the electron bunch has a homogenous, hard edge, charge distribution, $n(T)=n=$ constant.

And, since $b=c=$ bunch $\operatorname{radii}\left(r_{i}=r_{e}=2 \mathrm{~mm}\right), E_{e b}$ can be written as,

$$
E_{e b}=2 \pi e a b^{2} n x \int_{0}^{\infty} \frac{d s}{\left(a^{2}+s\right)^{3 / 2}\left(b^{2}+s\right)}
$$

Equation 2 has an analytical solution (see appendix A). If $a$ and $b$ are in centimeters, the integral in equation 2 equals $3.74 \times 10^{-6}$. The volume of an ellipsoid is given by,

$$
\frac{4}{3} \pi a b c
$$

And,

$$
\frac{4}{3} \pi a b c e n=Q_{e}
$$

Therefore, equation 2 becomes,

$$
E_{e b}=3.74 \times 10^{-6} \frac{3}{2} Q_{e} x
$$

Since equation 2 is in cgs units and $\mathrm{Q}_{\mathrm{e}}=10 \mathrm{nC}=30 \mathrm{stC}$, at the bunch edge $\mathrm{x}=150 \mathrm{~cm}$,

$$
\mathrm{E}_{\mathrm{eb}}=2.52 \times 10^{-2} \mathrm{stV} / \mathrm{cm}=7.57 \times 10^{2} \mathrm{~V} / \mathrm{m}
$$

Equation 3 gives the electric field at the apex of the electron bunch edge.

Now, the velocity of the most affected ions (those at the electron bunch edge) can be computed. Substituting from equation 3 into equation 1, for a gold ion at the electron bunch edge,

$$
\Delta \mathrm{v}=2.9 \times 10^{-2} \mathrm{~m} / \mathrm{sec}
$$


For a stationary ion in the bunch rest frame, this velocity change corresponds to a negligible energy change ( $1 \mathrm{eV}$ gold ion has a velocity of about $983 \mathrm{~m} / \mathrm{sec}$ ).

Even most affected ions seem to have a miniscule energy change. To ensure that it is not a result of miscalculating the (equation 2 integral) electric field, a simple calculation for the least affected ions is performed next. For ions at the edge of the ion bunch, the electron bunch can be approximated as a point charge of $\mathrm{Q}_{\mathrm{e}}=10 \mathrm{nC}$. In that case, $\mathrm{E}_{\mathrm{eb}}$ can be computed (in MKS units) using

$$
E_{e b}=\frac{1}{4 \pi \varepsilon_{0}} \frac{Q_{e}}{R^{2}}
$$

Substituting, for $\mathrm{R}=15$ and for $\mathrm{Q}_{\mathrm{e}}, \mathrm{E}_{\mathrm{eb}}$ can be solved to yield,

$$
\mathrm{E}_{\mathrm{eb}}=0.4 \mathrm{~V} / \mathrm{m}
$$

Repeating $\Delta v$ computations using equation 1 ,

$$
\Delta \mathrm{v}=1.55 \times 10^{-5} \mathrm{~m} / \mathrm{sec} \Rightarrow \approx 0 \text { energy change. }
$$

\section{Ion Energy Spread Caused By Movement Through Bunch Potential Well}

In the bunch rest frame, there is a relative motion between a number of ions and the electron bunch. Consequently some ions can undergo an energy change, if their relative motion during the interaction period results in movement through a potential difference that is generated by the electron bunch. As a consequence of the conservative nature of the interaction, no net energy exchange can occur. However, larger ion energy spread could result. Figure 2 is a rough illustration of the electric potential $\mathrm{V}$ inside the ion bunch along the $\mathrm{x}$-axis (direction of bunch motion). Ions, whose relative position changes from $\mathrm{A}$ to $\mathrm{B}$ and vice versa during the interaction period, undergo maximum energy change. 


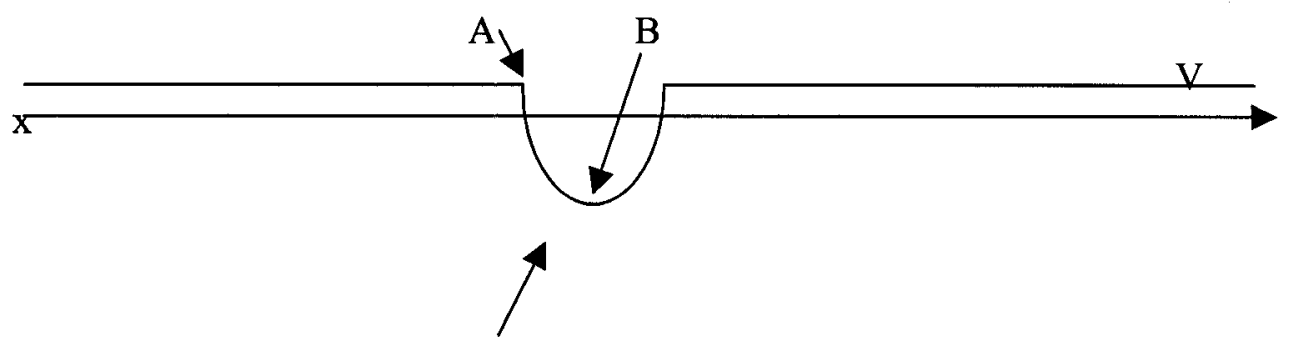

Electron Bunch Location

Figure 2

In addition to being in "proper" location in configuration space, ions must have a relative velocity that results in a 1.5 meter location shift to undergo maximal energy change. Since the interaction period is $1 \mathrm{nsec}$, their relative velocity should be $1.5 \times 10^{9} \mathrm{~m} / \mathrm{sec}$ (greater than $\mathrm{c}$ !); there are no ions that can undergo maximal energy change. And, since the ions have $\Delta \mathrm{E} / \mathrm{E}$ of about $3 \times 10^{4}$, there are most likely ions that can undergo some energy change.

Even though ions can undergo less than maximal energy change, that energy change is, nevertheless, calculated to show that any energy change that results from this effect is miniscule. To compute mid-to-edge (of electron bunch) potential difference, an expression for interior potential $V_{i}$ of a uniformly charged ellipsoid from Kellogg's book, ${ }^{2}$ can be used. To have consistent notation, this expression can be written as,

$$
V_{i}=-A x^{2}-B y^{2}-C z^{2}+D
$$

Where,

$$
A=\pi a b c n e \int_{0}^{\infty} \frac{d s}{\left(a^{2}+s\right)^{3 / 2} \sqrt{b^{2}+s} \sqrt{c^{2}+s}}
$$

And,

$$
D=\pi a b c n e \int_{0}^{\infty} \frac{d s}{\sqrt{a^{2}+s} \sqrt{b^{2}+s} \sqrt{c^{2}+s}}
$$

Along the $\mathrm{x}$-axis, $\mathrm{y}=\mathrm{z}=0$. The potential difference $\Delta \mathrm{V}_{\mathrm{i}}=\mathrm{V}_{\mathrm{x}=0}-\mathrm{V}_{\mathrm{x}=\mathrm{a}}$, where $\mathrm{x}=0$ is the electron bunch center. Thus with equations $2,5,6$, and $7 \Delta \mathrm{V}_{\mathrm{i}}$ becomes,

$$
\Delta \mathrm{V}_{\mathrm{i}}=\mathrm{E}_{\mathrm{eb}} \mathrm{a}
$$

Hence, from equation 3 , maximal ion energy change possible $\Delta \mathrm{E}$ is 


$$
\Delta \mathrm{E}=\mathrm{q}_{\mathrm{i}} \Delta \mathrm{V}_{\mathrm{i}}=\mathrm{q}_{\mathrm{i}} \mathrm{E}_{\mathrm{eb}} \mathrm{a}=1.4 \times 10^{-14} \mathrm{eV}
$$

In fact, there might be ions that can move from a location outside the electron bunch to the electron bunch interior. Those ions could gain an additional miniscule energy as they move to the electron bunch edge from a location in the ion bunch (based on equation 4).

In principle parallel ion energy spread calculated in this note should pose no problem, since $\Delta \mathbf{E}_{\max } \ll 1 \mathrm{eV} \ll \Delta \mathbf{E} / \mathbf{E} \leq 3 \times 10^{4}$.

\section{Remarks}

Ion space charge was neglected. Its inclusion would have slightly reduced the calculated energy spread. But, the effect is indeed negligible.

Actual charge distribution is peaked. Hence the electron bunch potential well is deeper, yielding somewhat larger ion energy spread. However, $\Delta \mathrm{E} / \mathrm{E}$ of $3 \times 10^{4}$ corresponds to ion energy spread that is in the GeV range. There is nothing to suggest that $\Delta E_{\max }$ can even approach that range. Furthermore, due to kinematic compression ${ }^{4}$ effects, existing energy spread in the bunch rest frame is larger than what $\Delta \mathrm{E} / \mathrm{E}$ in the lab frame implies.

\section{Appendix A}

Assistance in computation from Mike Blaskiewicz and Alessandro Ruggiero is gratefully acknowledged. In equation 2 , the integral I

$$
I=\int_{0}^{\infty} \frac{d s}{\left(a^{2}+s\right)^{3 / 2}\left(b^{2}+s\right)}
$$

has an analytic solution. ${ }^{3}$ In one of its forms it can be written as,

$$
I=\frac{2 a\left(\sqrt{a-b}-\sqrt{a} \operatorname{ArcTanh}\left[\frac{\sqrt{a}}{\sqrt{a-b}}\right]\right)}{\sqrt{a^{3}}(a-b)^{3 / 2}}+\frac{\pi \sqrt{-\frac{1}{b-a}} \sqrt{a-b}}{a \sqrt{a-b}-b \sqrt{a-b}}
$$


Using Mathematica $\otimes$ in Alessandro's PC, $I=3.74 \times 10^{-6}$ for a \& b in centimeters and 3.74 for $\mathrm{a} \& \mathrm{~b}$ in meters. To confirm results, equation 2 was converted to MKS. With $\mathrm{I}=3.74$, $\mathrm{E}_{\mathrm{eb}}=7.57 \times 10^{2} \mathrm{~V} / \mathrm{m}$ was obtained again.

Independently, Mike simplified I into a form of

$$
G(r)=r \int_{0}^{\infty} \frac{d u}{(1+u)^{3 / 2}(r+u)}
$$

Where $r=b^{2} / a^{2}$. With his own program, Mike generated a plot of $G(r)$ versus r. Reading (eyeballing) the value $\mathrm{G}(\mathrm{r})$ for the appropriate values of $\mathrm{a} \& \mathrm{~b}$ (dimension of the electron bunch ellipsoid), a value of $E_{e b}$ that is in good agreement (within 15\%) with Mathematica ${ }^{\circledR}$ results is obtained. Hence, there is a reasonable level of confidence in the results.

References:

1. F. J. Sacherer, PAC 1971, pp. $1105-1107$.

2. O.D. Kellogg, “Foundation of Potential Theory” Springer - Verlag Publications, New York, 1967 Edition.

3. See for example I.S. Gradshteyn and I.M. Ryzhik, "Table of Integrals, Series, and Products" Academic Press New York.

4. Effect is well known in optics, e.g., S.L. Kaufman, Opt. Comm. 17, 309 (1976). 